Positive; antitoxin; recovery; wore tube seven days; age 5 years.

Dec. 13, 1897. Courtesy of Dr. A. K. Worthington. Positive; antitoxin; recovery ; age $21 /$ years; wore tube six days. Dec. 14, 1897. Courtesy of Dr. H. C. Snitcher. Positıve; antitoxin; recovery; age 13 months; wore tube nine days. This was the four th case of diphtheria in the family, and the second one operated upon.

Dec. 21, 1897. Courtesy of Dr. Frank Dulin. Positive antitoxin; recovery ; age 6 years; wore tube five days.

Dec. 30, 1897. Courtesy of Dr. C. P. Perry. Positive ; antitoxin ; recovery; age 2 years; expelled tube on fifth day.

Jan. 1, 1898. Courtesy of Drs. MeLaughlin and Weaver. Positive; antitoxin; recovery; expelled tube on fourth day age 6 years.

Jan. 15, 1898. Courtesy of Dr. J. S. Neva. Poeitive; anti. toxin; recovery ; age 6 years ; tube worn five days.

Jan. 31, 1898. Courtesy of Dr. P. V. Carlin. Positive ; antitoxin ; recovery ; age 21/ years. Tube removed on flfth day.

Feb. 3, 1898: Courteey of Dr. P. V. Carlin. Positive; an toxin ; recovery; age 6 years; removed tube on fourth day.

Feb. 15, 1898. Courtesy of Dr. P. V. Carlin. Positive antitoxin ; recovery ; age 8 years ; removed tube on fourth day.

Feb. 20, 1898. Courtesy of Dr. D. R. Lucy. Positive; antitoxin; recovery; age 2 yeare; removed tube on sixth day.

Mar. 3, 1898. Courteey of Dr. I. B. Perkins. Age 17 months ; positive; antitoxin, five doses, each 1000 units; recovery; re moved tube on fifth day.

Apr. 27, 1898. Courtesy of Dr. T. J. Carlin. Positive; anti toxin ; recovery; removed tube on fifth day ; age 2 years.

May 11, 1898. Courtesy of Drs. Ellsner and Gorsuch. Posi tive; antitoxin; recovery ; removed tube on flfth day; age 6 years.

May 12, 1898. Courtesies of Drs. Warren and Worthington. Positive; recovery; removed tube on sixth day; age 2 years.

May 24, 1898. Courtesy of Dr. P. V. Carlin. Positive antitoxin ; recovery ; tube ejected two days after introduction age 20 months.

In this connection I would allude to an adult, 40 years of age, with diphtheritic croup, to whom I was called in consultation with Dr. W. E. Shotwell. The patient expelled a membranous cast of the trachea and recovered under repeated doses of antitoxin without an operation. Recovery was formerly so very rare in the adult, that I believe this case is worth recording. I should also call attention to a case to which I was called by Dr. P. V. Carlin, March 1, 1898, and which has been excluded from this report, as the patient was moribund and died during the operation, and could not be resuscitated, although tracheotomy was also performed. This is the only case that has been excluded.

To recapitulate: of the thirty-eight cases operated upon, five were under 2 years of age with four recov. eries, or 80 per cent, eleven were 2 years old, with eleven recoveries, or 100 per cent.; six were 3 years old, with six recoveries, or 100 per cent.; seven were 4 years old, with six recoveries, or 85.7 per cent.; two were 5 years old, with two recoveries, or 100 per cent.; six were 6 years old, with six recoveries, or 100 per cent., and one 8 years old, with one recovery, or 100 per cent.; total, thirty-eight cases, with thirty-six recoveries, or 94.7 per cent. - a mortality of only 5.3 per cent. in a class of cases that formerly was dreaded above all others.

A few years since it was my experience, out of several hundreds of operations to have saved 35 per cent. Why this great reduction in mortality from 65 per cent. to 5.3 per cent.? There is but one answer. It has been due to the use of antitoxin. If my experience stood alone the conclusion might well be doubted, but inasmuch as the great reduction in mortality has been noted by all operators, it must be conceded to be correct. In former years patients died, in the great majority of cases, after intubation or tracheotomy, from the extension of the diphtheritic exudate into the bronchi. At present this rarely occurs. I am fully convinced that when antitoxin is properly and energetically employed in full doses and repeated, the disease is at once cut short and no further progress occurs. I am also convinced that if a patient dies after intubation from bronchial obstruction due to the presence of diphtheritic exudation, the remedy has either been used late, the extension having taken place before its administration, or it has been used with a hesitating hand and in insufficient dosage, or the preparation has been of uncertain strength. When a chld is taken ill with diphtheritic or membranous croup-which I consider identical-the danger is so great that hesitation in the heroic use of antitoxin is almost criminal. I certainly should advise a full dose of 2000 units even for a child, and half the strength for infants, repeating the dose in twelve or sixteen hours. In one of the cases reported, four doses of 1000 units were given an infant 13 months old, and in another 17 months old, five doses of 1000 units without any injurious effect.

Even at this late day we occasionally meet with those who still cling to former methods of treatment, hoping to carry their patients through without subjecting them to the dangers (?) of antitoxin, and who are apparently afraid of the remedy and hesitate about repeating the dosage. I would again and again repeat and emphasize the fact that antitoxin must be given early. It must be given in full doses and repeated once, twice or thrice, if necessary. My confidence in antitoxin is so great that I sincerely believe that with intubation, aided by the proper and free use of this remedy, death from diphtheritic croup should rarely occur.

\section{DIPHTHERIA AS VIEWED BY THE GEN- ERAL PRACTITIONER DURING THE LAST YEAR.}

Presented to the Section on Diseases of Children, at the Forty-ninth Annual Meeting of the American Medical Association. held at Denver, Colo., June 7-10, 1898.

BY ALEX. MCALISTER, M.D.

CAMDEN, N. J.

It is not my aim to present a learned dissertation either on the pathology or scientific treatment of diphtheria. These subjects have been before the profession so constantly during the last three years that such a paper would prove only an intrusion upon your valuable time. What $I$ wish to do is simply to make a few practical observations that must be patent to every general practitioner. Until recently diphtheria ranked as the gravest of the anginas. and the most dreaded of the acute febrile affections. This applies with especial emphasis to the laryngeal types, formerly largely regarded as a distinct disease, but now generally recognized as identical with diphtheria of the larynx and properly treated with heroic doses of antitoxin. Yet the nasal and naso-pharyngeal types with their preponderance of constitutional manifestations and former frightful mortality were scarcely less dreaded both by the physician and parent.

Recent advances in diagnosis and treatment, it seems to me, have left scarlet fever at the head of the anginas and typhoid at the head of the acute fevers in point of morbid possibilities. Even the progress which has been made in diagnosis is to be credited to antitoxin, it being due to the new impulses contributed by serum therapy. In the American Pediatric Society's report on the collective investigations of the 
antitoxin treatment of laryngeal diphtheria in private practice, this advance is admirably noted in the following words: "In the early days of intubation it was customary to speak of the percentage of recoveries, and 24 to 27 per cent. were considered good result. In the last report the recoveries had crept up so high in the one hundred cases that it seemed more natural to speak of the percentage of mortality"; and again, in speaking of $O^{\prime} D$ wyer's personal experience prior to the introduction of antitoxin, in nearly five hundred operative cases of laryngeal diphtheria in which the mortality rate was 72.44 per cent.: "When he had reached the seventieth case on the fifth hundred something occurred which carried the phraseology up over the divide. At this point in history, antitoxin arrived and interrupted forever the old series. In his next fifty-nine cases the mortality was 23.7 per cent."

Recognizing that the past year was doubtless exceptional in many a section of our country for the small number of cases of diphtheria which appeared, it may nevertheless be observed that, incidentally with the more general introduction of serum therapy, we have a decrease in the number of cases as well as in the severity and popular dread of the disease and the rate of mortality. This wholesome change is due to an ever broadening and deepening conception of both diphtheria and its biologic antidote, antitoxin.

The resort to immunizing injections to prevent the spread of diphtheria to other members of the family where a case or two has appeared, is not so largely practiced as the value of the measure demands, but is rapidly gaining in popular favor. But aside from any resort to this measure, it is evident that serum therapy has exerted a most potent influence in restricting the number of cases. While it is always within the range of possibility for the most malignant case to follow the mildest case, yet the proneness to spread the infection is always greater in severe cases. Admitting this, it is clear that whatever checks malignancy also controls the tendency to spread and materially restricts the number of cases that would otherwise occur. This beneficent influence can never be adequately estimated, although it is very considerable. One of the most encouraging experiences of many a general practitioner who has adopted the serum treatment of diphtheria, is that he now only occasionally meets more than the initial case or cases in a family. Many of the latter class even assert that they never even meet the exceptional new infection.

In view of all these facts, it would seem that the attitude of the general practitioner toward the serum treatment is a more seasonable subject for discussion than the merits of antitoxin. In the investion of this most interesting subject the initiative was doubtless taken by Dr. George L. Cole of Los Angeles, Cal., who made a study of the question in his own State and reported, recently, in an admirable paper entitled "Sero-therapy," before his State Medical Society. In reponse to a circular letter addressed to about three hundred physicians he received replies from 156. Of this number, only four expressed themselves as antagonistic to serum treatment, but none of these had personally tried the remedy nor had a desire to do so. With the opportunity to compare the clinical notes of hundreds of physicians who have each treated many cases in private and hospital practice, of thousands of others who have less numbers, and with the unequivocal results of great collective investigations con- ducted both at home and abroad, it would seem that even the most obscure general practitioner is no longer justifiable in refusing to give his diphtheria patients the full benefit of the more humane and successful serum treatment. According to my personal observations, this effect is rapidly being attained. Physicians who were once loud in their opposition are now warm advocates, or in preference to the humiliation of a public retraction, have become secret users of antitoxin.

Speaking of the product, as now freely available to every physician, it is to be observed that marvelous progress has been achieved. I was one of the first in the East outside of our large cities to employ the remedy. That was before antitoxin was prepared in Philadelphia for commercial or other purposes. The article was then difficult to procure and very expen. sive. The quantity of serum necessary to inject in order to secure what was then regarded a full curative dose was inordinate, being upward of one fluid sunce. The syringe which I then employed has long since been laid aside as suggestive rather of minor rectal surgery. The improvements which made it possible to inject much larger doses in very much smaller quantities of serum, that is to say, which gave us concentrated serums, are without parallel elsewhere in the pharmaceutic sciences.

One year ago the most concentrated product in the market contained 500 immunizing units to each cubic centimeter of serum, but at present serums are avail. able of 1000 and even 2000 units per cubic centimeter. The latter, it seems to me, have reached a degree of concentration which is nolonger advantageous or even practical. A serum which contains nearly $150 \mathrm{immu}$ nizing units to each minim of the serum, or even half this strength, is relatively difficult to handle, owing to the small bulk and the unavoidable source of loss. while it remains a question whether such high concentrations possess any special therapeutic advantages. at all. It seems to me, and in this $I$ voice the sentiment of the general practitioners with whom it is $\mathrm{my}$ pleasure to exchange views from time to time, that a product permitting the administration of 1000 units of antitoxin in from 2 to 4 centimeters, i. e., from onehalf to one fluid dram of serum, if well ripened and. conveniently bottled, is the ideal antitoxin from every point of view. It is conceded that the more concentrated the product is the more readily deterioration takes place, so that the higher strengths are relatively unreliable after one to four months. This in itself is a considerable disadvan tage to the general practitioner, who, more often than any other class of physicians, finds it indispensable to keep an available quantity of a strictly reliable product constantly upon his shelves. To him there are a score of elements that enter into a particular antitoxic serum of vastly more importance tnan higher degrees of concentration, per se, however commenduble as an achievement in its preparation the latter may be. Chief among these elements I would name a high unit value, seasoning of the serum, a definite system of standardizing, con-venient and sufficiently strong bottles and uniform potency.

DISCUBSION OF DIPHTHERIA.

Dr. Louis Fischer, New York City-In the paper of Dr. Engleman this morning I was interested in hearing that her results with the mixture of the antitoxin with anti-streptococcus serum were good. I was very much interested recently to hear from some gentlemen diseuesing the subject, that they: 
endorsed the view I maintained, namely, that antistreptococcus serum today can not be classed as a remedy to be used. My experience dates back but two years, when I had the pleasure of seeing a series of cases under Dr. Baginsky of Berlin. We did not find it of value. In one case, in which the antistreptococcus serum was used, the bad effects were due to its use. Today we have not a streptococcus antitoxin, but only an antistreptococcus serum, which is not equally of value in all cases. While doing good in erysipelas, it does not do good in cases of scarlet fever, although we have proven that the streptococcus is the etiologic factor in scarlet fever. Therefore, I wish to reiterate what I said in New York, that I believe the time has not come when we may say that we are permitted to use the antistrepticoccus serum in the same sense that we use the antitoxin in diphtheria. I would be pleased to hear if the various authors who have read today will state whether they have used this with the antitoxin, and their success.

Dr. Engleman stated that she fully believed the etiology to be still in a confused state. Mr. Chairman, I can only tell you of a very interesting case of a child who had an affection of the throat and showed the Klebs-Löffler bacillus. A child brought into the hospital for the relief of a phimosis, was operated upon and some of the smegma inoculated in a test tube. When placed under the microscope beside a culture obtained from a case of diphtheria, a friend of mine, who is a bacteriologist, was absolutely unable to differentiate them. When in Berlin I saw the streptococcus and staphylococcus in a great many cases. While the accepted theory is that the Klebs-Löffler bacillus is the sole cause of diphtheria, and that it can produce diphtheria, I think there is still room for im provement in the study of the etiology of diphtheria. I learned from another bacteriologist, who said he knew of the bacillus of smegma, that is similar to the diphtheria bacillus. I need only say a word of the pseudo-diphtheria bacillus, which is frequently present under normal conditions.

Passing to Dr. Jerowitz' paper, the gentleman referred to the fact that he does not use iron. I was rather surprised to hear that statement, because I regard iron as one of the most important drugs in the treatment of diphtheria. While the accepted conclusions in regard to antitoxin are so positive, I believe iron etill holds its own as a remedy in diphtheria. The treatment of diphtheria was in such a condition of chaos four or five years ago, in regard to this antitoxin question, that $I$ believe we are to be congratulated on the progress made in this line, and I believe it is due largely to some men who have gone through. out the country and advocated the use of antitoxin. Largely it is due to such work as that of Dr. Rosenthal. I will state, however, that I do not use antitoxin in every case. It was only about two months ago that Dr. Garrigues telephoned me to bring enough antitoxin for four cases. But I found only some little spots, which by a little gargling got well. I know another case in which by using a little wash the child recovered. I do not use the antitoxin unless there is no improvement in twelve hours. However, if I am called to a case in which a whole tonsil or the palate is involved or when there is stenosis, I would regard it as criminal not to use antitoxin. I never give under two thousand units of antitoxin in any case.

The paper of Dr. Waxham is a valuable contribution. The statistics are such that it would be a crime not to use antitoxin where there is laryngeal involvement. The Doctor has shown the old tubes of O'Dwyer. We have had the rubber tubes introduced; I believe I showed some of the tubes last year at the meeting of this Section in Philadelphia, and we have been using them since. The lime salts do not deposit on them, which is of value especially in the cases where we must use the tubes for a long time. In the case of a child I intubated on February 20, I am confident if I had used a metal tube I would have had to use a series of tubes by this time, but the rubber does not permit the deposit of lime salts on the surface. Dr. O'Dwyer's new sets have also these new tubes and they are made according to his own directions. The tubes I use are made corrugated, because I find them so slippery that they are coughed out if we do not have them corrugated. The gag of Dr. Waxham I have used and I believe it is an excellent gag. I should like to ask Dr. Waxham how he treats his prolonged cases of stenosis. Some cases were reported by Dr. O'Dwyer at the last meeting of the Pediatric Society. I refer to the class of cases where you have intubated and left the tube in for a week or ten days, removed the tube and have stenosis, and must they have prolonged intubation? I have mentioned a case where the child has worn the tube over three months. I have tried tracheotomy in several of those cases and $I$ have found that each and every one of them has died.

Dr. Ross Engleman, Chicago, in closing.-I have nothing to eay, except to emphasize the subject of polymorphism of the Löffler bacillus. As the Doctor says, I think the subject is still in an experimental state. I do not think we can say certain symptoms refer to the staphylococcus or streptococcus or Löffler bacillus infection, because of this polymorphism, and we often do not get anything except a mixed infection. In my series of cases I did not use the antistreptococcus serum in sufficient doses, so I would not express an opinion as to its use in these cases. As to the question of the prolonged intubation, I am very glad to hear from Dr. Fisher of the use of the rubber tubes. I have had a few cases that were very troublesome and shortly after I had those cases I read O'Dwyer's article, I think in the July number of the Archives of Pediatrics, in which he referred to the use of alum and paraffin, which seemed very ingenious and with which he said he cured his ulcers in a short time where the tube had been worn three months or longer.

Dr. JAQUES, Chicago.-I had charge of the South Side Dispensary in Chicago at the time Dr. Waxham was beginning his career as an intubator and I have called him out, in the most bitter and disagreeable weather, and he never has failed to respond to perform intubation. In nine out of ten of those cases death followed. When I recall the experience he went through I am glad no such experience will be possible for the next generation. We should have in mind always the fact that it takes two things to make a case of diphtheria: First, the predisposition to the disease; second, the presence of the germs. I have frequently obtained cultures of the Klebs. Löffler bacillus from my mouth, although I have never had diphtheria in my life. In any individual not susceptible to diphtheria, there is, through the action of the cells, an antitoxin produced which protects the individual from the action of the germs. When the individual loses this condition of resistance or it falle below a certain standard and the KlebsLöffler bacillus is introduced on the membrane, Klebs-Löffler diphtheria will follow and will progress according to the susceptibility of the individual. Unless you have the clinical symptoms following the finding of the germ, you do not have diphtheria. Dr. Waxham, in his paper, stated that he always gave at least two thousand units to a child and more to an adult. If you will pardon me for a moment, I wish to illus. trate the stand I have taken, that the younger the child the larger the dose of antitoxin should be, not in the amount of serum but in units. We understand that the growth of the bacilli produces the ptomains. In the experiments we estimate the amount of toxin which kills the animal by the size of the animal, and the smaller the animal the smaller the amount of toxin required to produce the animal's death. The growth of bacilli here is as large in the infant as in the adult, and therefore a larger amount of antitoxin is required. I use three or four thousand units in infants. I want you to beware of the concentrated antitoxin. A drop will stay in the syringe, a drop will be lost, probably, in seeing that you do, not inject 
any air. I prefer the antitoxin of average concentration, say $5 \mathrm{cc}$. to three or four thousand units. One of the gentlemen who read a paper said he did not think antitoxin is a preventive of paralysis. I do think the gentleman has carefully considered that statement. You might as well say that water does not prevent the spread of fire. Antitoxin does prevent paralysis. The toxin produced' by the Klebs.Löffler bacillus causes necrosis, and when that ptomain accumulates to a sufficient amount to produce destruction of the corpuscles of the blood, the kidney tubules and the destruction of the vital centers, the patient will die. So if you give your antitoxin it must be given before this destruction occurs and then you will prevent paralysis.

Question.-I would like to know how long they quarantine patients to prevent the spread of the disease.

Dr. JAQUES-In the Health Department of Chicago we do not placard any house unless the Klebs-Löffler bacillus is found in the case and the diagnosis is made by one of the inspectore in the health department. We have not followed the quarantine very carefully for the reason we work there in harmony with the physicians. We are simply the assistants of the physician. We say to the doctor, you go to your patient, take with you a little culture material, give it to us after inoculation and it will be returned to you as your private property. The patiente do not know in one-fifth of the cases that the health department had anything to do with the case. The physician is responsible for the spread of the infection, and he looks after it then very much better then the health department could do. As a result our diphtheria statistics, as perhaps you know, have been remarkably low, being in the charity cases only 6.7 , when formerly they were nearly 40 .

Question-How long do you keep the house placarded?

Dr. JAQUES - We leave that to the discretion of the Doctor. Often it does not stay on the house over an hour and often it remains on the house for a long time. The patient is at once isolated, and if the patient is isolated and put in the care of a trained nurse, the individuals in the house are in no more danger than an individual in an ordinary street car. If the physician desires to take the responsibility of the case, the placard need not go on the house at all. Every doctor knows that when he calls on the health department he will not be interferred with in any way. If the patient is so poor that antitoxin can not be bought, it will be furnished them by the health department without cost.

Question-I would like to ask the physician from Chicago, if when he found the Klebs.Löffler bacillus in his throat, and thought he was not subject to diphtheria, did he not think he was a menace to other persons, who were debilitated?

Dr. JAQUES-I think the only time I would be a menace would be when kissing, and I am not subject to that habit. When I found this bacillus in my throat, I was very careful to take means, by gargling, to keop my throat from being a subject of infection. I have gone from a case of intubation to a case of confinement and performed version and do not know that I ever transmitted diphtheria. There is a great mistake in the belief among the general public as to the contractibility of the disease and the danger of physicians. If they are clean, there is very little danger of contagion. Contagion is spread only by contact and not at all through the air.

Question-Do you ever fumigate the premises after a case of diphtheria?

Dr. JAQUEs-We have spent several thousand dollars in the last year in the practical methods of fumigation, and we have found formaldehyde gas one of the most practical methods. It is clean, leaves no odor and is one of the simplest and best methods. We simply take formalin, or the 40 per cent. solution of formaldehyde gas, add four parts of water, stop up the cracks in the room, extend a sheet across the room and throw the solution on the sheet. We then get out of the room as quickly as possible. The disinfection is perfect. We place tubes, wrapped in flannel, in various places in the room and if any of them grow, the disinfection is repeated. This does away with the use of sulphur and we find it by far the best method of disinfection.

Dr. Agney, Philadelphia-I think Chicago does better than Philadelphia. In the first place, we have a hard and fast law that all cases have to be placarded. When the culture is made the house is placarded and the placard is removed when disinfection is attended to. I believe one of the causes of spread of the disease is the non-report of cases of diphtheria, due to the fact that the placard interferes with people's business. I think, myself, there ought to be discrimination and where the physician in charge is willing to assume the responsibility of isolation, the placard should not be on. But the act of 1895 states that a placard shall be put on, and the Board of Health of Philadelphia, who have the discretion in this, have insisted on this placard being put on every house. One of our most distinguished physicians in Philadelphia un. fortunately had a case in his house, that I am satisfled every. thing was done to isolate, but in that case the placard was put on his house. I believe cases of diphtheria can be so taken care of that the risk to other people will be reduced to the minimum, probably reduced to nothing. It would be interesting to know whether in Chicago they use force to remove patients, where the doctor does not assume control of the case. We have to deal with a population in my city where no isolation is used and we are allowed to send a force in and taike the patient out. You will all admit that under such conditions a case taken to our municipal hospital removes the center of contagion. There is no question, when such a case is removed, that a benefit is conferred upon the city. I know, myself, of a case which was not reported, that was visited by children invited from a kindergarten and several lives were lost and several children were eick for a long time. I believe at least a hundred cases were kept out from the epidemic traced to this case. The case was not reported and the doctor was brought into court and fined $\$ 5$ for I don't know how many lives and how many cases of diphtheria.

Dr. W. H. Setbert, Pennsylvania-We have had the pathology, symptomatology, treatment, preventive measures, and even down to the police regulations, but one point has not been mentioned. I refer to diphtheria occurring in regions not accessible to view, as in the alimentary canal or possibly in the posterior nares, where, however, it might be seen by proper instruments, and in other regions. The Doctor has stated that he has gone from intubation cases to obstetric cases without danger. Recently, I had some experience where a consultant, who had been treating malignant cases of diphtheria, was called to help in a case. I asked him to examine the case, which he did. He did not speak of his diphtheria case until the after-course of our case of obstetrics. There were peculiar symptoms appeared. In fact, there were the general symptoms of diphtheria, but there were no symptoms of diphtheria about the throat. The main symptom was complete anorexia, absolute repugnance of food. That has not been referred to but is usually understood. On examining everywhere, carefully, I could find nothing to indicate diphtheria until with the speculum I examined the vagina. On the fifth or sixth day we found a diphtheritic patch inside of the vagina, which was nearly an inch in diameter. The case occurred before the introduction of antitoxin, and the patient went on and died from diphtheria. A few years ago, J. Lewis Smith reported a case which died from what he had treated for some time for typhoid fever, but before death the patient passed a complete cast of the intestine, which proved to be diphtheritic on examination.

Dr. WeLCH of Philadelphia-It seems I find myself in the enemy's camp. That is to say, I stand alone in this matter. I 
have seen diphtheria, having had charge of a hospital where infectious diseases are constantly treated, for the past twentyeight years, and during the past six or eight years we have been receiving large numbers of cases of diphtheria, along with scarlet fever and some few of the other infectious diseases. The death-rate in our hospital before the days of antitoxin was never any thing like as high as has been stated here by some of the speakers today. Our death-rate before antitoxin was from 28 per cent. to 30 per cent., or along there somewhere as near as I can recollect now. And, gentlemen, it remains there today. We have not reduced the death-rate whatever. It is true in 1894 the death-rate was higher than ever; it was high all over the world. That is known by all who followed the literature of the period. The death.rate in our hospital was about 30 or 33 per cent. It was about the same in the Willard Parker Hospital of New York, and in the Metropolitan Hospital. However, in some of the hospitals the death-rate has been very high, as high as stated here today. After antitoxin came to our notice in 1894, we did our best to procure this agent, that our patients might have the benefit of it. We succeeded in the fall of that year and were able to give it to only sixteen cases, I think, in the latter part of 1894 . We watched those cases with a great deal of interest. Their temperatures were taken every hour or two, notes made every little while, and we had complete histories reported of the first five cases. They were pretty nearly the first cases in which antitoxin was used in Philadelphia. Dr. Fischer, who is present, knows something of those five cases ; two of them died.

We continued, and that year used antitoxin in about sixteen cases, but it was not lower than the usual death-rate. In 1895 it was not so difficult to procure antitoxin. The preparation of the antitoxin we used then was Behring's number two. It was not, of course, as easily and readily obtained in as large quantities as at present; it was high, and we economized its use as much as possible. I attempted to select the cases which $I$ thought most favorable to the action of the antitoxin. Everybody who had written on the subject said antitoxin was useful only when used in the early stages of diphtheria. I selected those that were from the first to the fourth day of the disease, well-marked clinical casses, some of them very severe and othere not so severe, but every one of them well-marked and in every one of them the Löffler bacillus was present on culture. Those which did not receive antitoxin were, first, exceedingly mild cases, which I believed would recover without any remedy; second, cases advanced beyond the fourth day, and third, malignant or moribund cases. It was thought they would serve no fair test of the remedy. At the end of the year in counting up the cases there were about three hundred received antitoxin and over five hundred did not receive it. The cases were not analogous and could not be compared. But the deathrate among those from the firet to the fourth day who received antitoxin was about 28.14 per cent., while among the other class it was 25 per cent. and a fraction, I think very nearly 26 per cent. Now, I do not say that these series of cases should be compared, for they are entirely different, as I have told you. I was surprised that the death-rate should be so high. In closely watching these cases after the administration of antitoxin, particularly my early cases, no one can well imagine my disappointment. I really expected antitoxin to do all they claimed for it. It did not. Now, I have no prejudice; I wish antitoxin would do what they have said it would. I thought I would get out of writing so many death certificates, but the patients have died just the same. In watching the cases I could not see such a beneficial effect from it. Sometimes we thought we did see the change in the exudate, that it assumed a more fat-like appearance and peeled off more readily. But right along the side of them you could pick out the same number that did not receive the antitoxin but behaved in the same way. I can not say that I have observed that the exudate dis. appears more quickly after the use of antitoxin. If there is any difference it is not striking. There are some cases where it peels off quickly, there are other cases where it remains for a long time, there are cases where it disappears and re-appears, and there are cases where it travelseven down into the larynx. A good deal was said about the temperature. Temperature in diphtheria counts for very little; except in the worst cases it is usually low. In the vast majority of cases the temperature is high at first, but in a short time it sinks to normal, or nearly so, and in bad cases to below normal. In the hospital you will see very few cases with high temperature, except in cases of broncho-pneumonia. This is very common and many of the intubated laryngeal cases die from it. Many of them die late; some die two or three weeks after they have been admitted into the hospital. A week or two after the tube has been removed bronchial pneumonia develops and they die. We all know the theory by which antitoxin acts, the theory of immunity, that in a person who has suffered from some one of the infectious diseases there is an antitoxin produced in the blood and that this antitoxin protects them against future attacks. This is a beautiful theory, but it seems to me it is not proved. No one has ever discovered antitoxin, described it or told us anything about it. Now, what does nature show us? What do we find in practice in our daily observations? Take, for instance, a woman who is rendered immune to smallpox, which is a common disease and one of the most contagious and infectious diseases we have. Take a person immune to smallpox, either by reason of vaccination or previous attack, say the individual is a female and there is an antitoxin developed in her blood which. protects her. That woman becomes pregnant and gives birth to a child. That child, it seems to me, should receive immunity from its mother. Yet that child, from the time of its birth, is susceptible to the disease. The woman may not be affected by a disease to which she is immune, but the fetus in utero may be susceptible. If it will protect her, why will it not protect the fetus in utero?

Dr. R. B. Gilbert, Louisville.-My experience has been somuch in common with that of Dr. Fischer that I regret the last speaker. I have treated cases without the eerum and have had a mortality of 65 or 70 per cent., and with the antitoxin I have had a mortality of from 7 to 10 per cent. The cases were in the same epidemic with the same environment and proper attention. I believe antitoxin should be used early and in the quantities stated by Dr. Fischer. In a case of laryngeal diphtheria in a child under two years of age it is scarcely worth while to use the serum late. But if we see such a case early we might have quite a success with it. My experience has been only in general practice through a number of epidemics and not in hospital practice. I have succeeded in saving a large number of cases, which I know would have died had I not used the serum.

Dr. Simmons of Nebraska.--I am sure antitoxin is as nearly a specific for diphtheria as any medicine we have for any other disease. I do not believe quinin is as much a specific for malaria as antitoxin is for diphtheria. In the early years of my practice I ran into a lot of diphtheria, in which all the phy. sicians lost nearly all of their cases. Nothing could save them. Twice in my medical experience 'since then, once about eight years ago and once about six years ago, I had the same class of cases. During the intervening periods we had a class of cases that were mild. I have been anxiously waiting with a good deal of fear for a chance to try antitoxin in the old type of diphtheria. About two months ago I had such a chance. Until then I was ready to say that I thought what I had read was all right, for I had used it for some years, but during the last two months I have had such experience that I do not care what statistics are brought up nor what anybody else may say, I know I have saved cases with antitoxin. I will just relate two instances in two families. One was a family of nine chil- 
dren. They were poor people and did not send for me or any physician until one of them was practically dead. Another case was in bed when I got, there. The glands of the neck were all swollen. The mouth and the fauces as far as I could see were covered with membrane. In a bed in the same room were two other girls, and from a room above 1 here were two boys coming down. In one of the cases there was evidence that it was going to be a very severe case. I did not use any antitoxin in the worst case because it was too far gone. To the others I gave antitoxin and I saw an immediate good effect. At the first sign of the membrane in the children not yet affected I gave immunizing doses of 500 units and they all got well. If you could have seen the cases with me, you would have believed as I do that those cases were saved by antitoxin. Across the street there was a brother-in-law who called a physician who is a bitter opponent of antitoxin. He did not use it and he succeeded in saving one of the four children. In one family the Christian Scientists created quite a rumpus but nothing was done to them. In that epidemic there was not a case died in which antitoxin was used and nearly all the other cases died. Therefore I feel like saying what I said two or three weeks ago in our local society, that I believe today a man is criminaliy negligent of his duty who fails to give antitoxin in diphtheria.

Dr. John A. Lockard-From my experience as interne in the New York Hospital, I wish to emphasize what has been said about the mortality. In eix months' experience there I have failed to see the membrane melt away any quicker with the antitoxin than without it. I have failed to see the so-called "black" diphtheria get well under the antitoxin treatment. In one class of cases $I$ saw a good result, and that was in the cases intubated. In its effect on postdiphtheritic paralysis I have seen no results you could call good. I have seen cases of heart failure with regurgitation just as marked and frequent. As to the antistreptococcus serum, we found that most of the incubated cases died from subsequent pneumonias. We took the cases with no physical signs of bronchitis on admission and injected the antistreptococcus serum, 2 c.c. every day. Out of the six cases we lost five. We found the rashes most confusing. The rashes of this and scarlet fever were so much alike that barring the throat symptoms they would be very confusing. I think, however, the statistics from the hospital are likely to lead us astray, especially in certain directions. Usually the cases sent in are old cases. The children have parente who are not particularly careful in observing the children and they do not recognize the disease until it is pretty well advanced. Another way in which the statistics may err is that we admit many cases as diphtheria which upon bacteriologic examination would be called a mere tonsillitis. In the children we do not have an opportunity to observe the melting of the membrane as well as in the adulte, because we were required to give antitoxin to all the children. But in adults it was optional, and we occasionally advised them not to take the antitoxin that we might observe the cases. Side by side, cases as near alike clinically as we could possibly get then, I have seen the membrane melt away without the antitoxin and others who had antitoxin remained in the hospital weeks longer. Another peculiar and significant fact, it seems to me, is that when our nurses become affected with diphtheria they do not use the antitoxin, and the same was true when my predecessor and successor had diphtheria.

Dr. H. D. Jerowitz, Kansas City, in closing-In respect to intubation, I might have had a great many more cases in my practice if I had been permitted to perform intubation when I wanted to, but as the majority of the cases were among ignorant people, they were very much afraid of mechanical treatment. Many of these cases presented cyanosis, upon whom I wanted to intubate, and the majority got well just the same. The difficult breathing seemed to be only transient. It shows the fact that a great many cases that seem to require intubation or tracheotomy at the moment you see them, may have the spell pass off in a few momints and the case for the time being will be better. Very often when these cases present urgent symptoms, the cyanosis and dyspnea are due not to the membrane, but more to the mucus, which may not be brought up a certain moment, and if an emetic is administered, or the child has a severe spell of coughing or crying, the urgent symptoms pass off and the necessity of intubation or tracheotomy is obviated. As to the use of ron, we all have our own opinions, which are based upon our own experience. I used iron in the early part of my practice, but $I$ never could see the rationality of it, nor could I see why it cures these cases. $\mathrm{Be}$ sides, my experience is that the cases that can be benefited by iron, can just as well get well with other treatment. I can not see what such an irritating remedy as the perchlorid of iron can accomplish in such a disease as diphtheria. It seems to be a very irritating drug, not only constringing the membrane of the throat, but of the stomach and bowels as well, thereby interfering with assimilation. I think the value of it is more from the chlorin, and for that reason I often use hydrochloric acid internally, expecting also an effect from it as it passes the throat. As to the antitoxin, the dose must be large. Two thousand units may be employed three times a day in laryngeal diphtheria. One gentleman referred to the fact the statement was made that antitoxin does not prevent paralysis. My paper did not say so. I said paralysis occurred in those cases that were either severe cases or cases of long duration. Those cases in which there was extension of the membrane or which lasted ten or fourteen days, were as a rule followed by paralysis. Then I further stated that in all cases where the antitoxin is used early, the membrane disappears in two or three days and it shortens the sickness, and from this fact it prevents paralysis. I stated that if the antitoxin was used early, no paralysis is found. Now, as to the epread of diphtheria and the placarding, it is very evident that there are a number of mild cases that do not go to bed and are not recognized, and children having a very mild form of diphtheria, that are hardly sick except possibly a slight headache, may go to school and spread the infection to others.

Dr. F. E. Waxham, Denver, in closing-In regard to the paper presented by Dr. McClanahan, I would agree entirely with his conclusions, so briefly and explicitly set forth. In regard to the paper presented by the first essayist, I would say that on account of my confidence in antitoxin $I$ have discarded all gargles, sprays, iron, and other remedies. I simp!y give a large dose of antitoxin, repeat it in ten or twelve hours, and give a third or fourth dose, if necessary. Antitoxin has been compared to vaccination. I think that is not proper at all. The vaccine disease is another disease; it is a disease, smallpox, modified by passing through the system of a cow. There is simply no comparison between antitoxin and vaccination. In regard to the health department, I was very much interested in the methods employed in Chicago. They are quite different from the methods employed here. Here the physician inoculates a test tube and takes it to the health department; if it is found to be diphtheria, the health department takes entire charge of the case. They isolate the case, and make repeated examinations of the patient's throat. As long as the case shows any bacilli in the throat, the case is isolated and the house placarded. If Dr. Jaques lived in Denver, he would not have the temerity to have the secretions of his throat examined. I am much surprised that any one, at this day, can oppose the use of antitoxin. I can not understand how any one, after reading the conclusions of the Pediatric Society, can fail to be convinced of the utility of antitoxin. After my experience in the treatment of diphtheria, I would feel that I was not doing my duty as a physician, not doing my duty to my profession, unless I advocated the use of 
antitoxin as the greatest remedy that has been presented to the medical profession during this century. I can not but believe there must be something wrong in the methods employed by those who have found so little benefit from its use. They either have not used the remedy early, or have not used the remedy in sufficient doses, or have not repeated it when necessary. I suspect the cases that have come under Dr. Welch's care are late cases, for frequently we know cases are sick three or four days before they are taken to a hospital. Then, if a bacteriologic examination is made before treatment is begun, another twenty-four hours is lost. I feel like agreeing with one of the epeakers when he says that he would feel guilty of criminal negligence if a case died under his care and he did not use antitoxin.

Dr. H. M. McClanahan, Omaha-I only wish to bear testimony that I believe most thoroughly in the use of antitoxin. I believe, however, we must not expect everything from this remedy. I believe the statement by Dr. Welch, that antitoxin can not undo the harm diphtheria has done, is true. I think the cases crowded together in the hospital will not do as well. But I think his line of argument should not give us reason to not use the remedy in privale practice. In the cases mentioned by Dr. Welch, I have no doubt he found mixed infection, over which antitoxin has no influence. I think we should get a clear idea of what this remedy should be expected to do, and what it should not be expected to do. I became thoroughly disheartened and discouraged with antitoxin. The patients would be relieved a few hours, and then the patch would extend on down. After the use of antitoxin in the past year I have intubated only twelve cases, and eleven of them have recovered. I can go back five years, when the patients under tracheotomy or any other treatment that we had, gave a mortality of 50 or 75 per cent. It would be a retrograde step if we were to allow ourselves to lose our faith in the use of antitoxin. But we should not expect it to do everything. When your examination shows that you are dealing with a mixed infection, then let us not stop with the use of antoxin. Then $I$ believe nascent oxygen is of benefit. Even some of those cases can be saved by the use of nascent oxygen with antitoxin. In one case I saved a child in this way when the lips were blue. It leads me to believe I will never give up a case of diphtheria until the case is cold and stiff and dead. I have the most profound belief in the value of antitoxin in neutralizing the toxins of diphtheria.

\section{THE EARLY DIAGNOSIS OF DIPHTHERIA.}

Read before the Mississippi Valley Medical Association, Oct. 13, 1898. BY WILLIAM K. JAQUES, MD.

DIRECTOR ANTITOXIN STAFF HEALTH DEPARTMENT CITY OF CHICAGO.

Outside of laryngeal complications, the mortality in diphtheria is due to the toxin produced by the Klebs-Loeffler bacilli. No physician can successfully treat diphtheria unless he understands the nature of this toxin, how it is produced, and how the cells may be fortified against its destructive action. He must understand that the Klebs-Loeffler bacillus is a distinct living entity or vegetable organism; that one of the products of its existence is diphtheria toxin, just as the result of the life of the yeast plant in alcohol.

Other germs in anginas will cause inflammation, pain, temperature and membrane, but only the KlebsLoeffler bacilli produce the deadly toxin which begins with their invasion and progresses to a fatal amount as swiftly as conditions permit.

To appreciate the danger of his patient, a physician must understand the rapidity with which these bacilli multiply under favorable conditions. The clinical symptoms manifesting their residence may give no indication as to the rapidity with which the fatal amount of toxin is being produced. The best means of comprehending this fact is to use the microscope and follow the multiplication of the KlebsLoeffler bacilli in their cultural life. Inoculate a box of culture-medium with infected mucus and place in an incubator at body temperature. At the end of three hours, a cover-glass pressed upon the surface, properly stained and mounted, will show sufficient bacilli, if they are present, to give an idea of the number and arrangement of germs upon the surface of the medium. Incubate the same culture eight hours, and an examination will show that the germs have multiplied many times. Eighteen hours will reveal a large and complete colonization, from which an idea can be gained of the way in which the KlebsLoeffler bacilli multiply under favorable conditions. Understanding, as we do, that toxin is a product of these germs, their multiplication means an increased amount of toxin, which soon reaches the fatal point unless checked by the use of antitoxin. This demonstrates the importance of a physician knowing at the earliest possible moment what germs are present in an angina. It may be said that in the majority of cases there are two things he does not know: the extent of the infected area, and the resisting power of the patient.

The diagnosis of diphtheria in the early stage of the disease must be made from both clinical and bacterial evidence. From his patient the physician finds clinical manifestations of an invasion of the mucous membrane of the respiratory tract. When his microscope reveals the germs that are causing this invasion, he can then say whether or not the disease is diphtheria. Delay in ascertaining this information can only be done at great risk to the patient's life and the doctor's reputation:

In order to ascertain the value of direct diagnosis, -by which we mean the examination of material taken directly from the infected area without waiting for incubation--the Chicago Health Department has introduced the following culture outfit for the use of physicians; a sterilized swab is placed in a glass tube; a slide carefully wrupped in paper is placed with this in an envelope together with a culture box and directions for using same. Physicians are requested to inoculate the swab from the inflamed site, spread a little of the mucus upon the slide and allow it to dry. The culture medium is inoculated from the same swab and it is returned to the glass tube. The whole outfit is then to to be sent to the nearest incubator station or laboratory.

As this method has been in use but a short time, its value has as yet to be demonstrated conclusively. From my own experience I have received very valuable information. In one case I did not find any KlebsLoeffler bacilli in the culture medium but found that a large colony of the bacilli had incubated upon the mucus which was still left upon the swab. In a num. ber of cases I have been able to make a direct diagnosis by staining and examining the slides as soon as they come to the laboratory. When no antiseptic treatment has been administered before the culture is taken, and the disease manifests malignancy, by stupor, hoarseness or swelling of the cervical glands, it has been possible in about 50 per cent. of the cases to find sufficient bacilli to warrant a diagnosis of diphtheria, sometimes before any trace of membrane is visible. When it has been possible to get a small 